REVISTA DE GEOCIÊNCIAS DO NORDESTE

Northeast Geosciences Journal

v. $7, \mathrm{n}^{\circ} 1(2021)$

ISSN: $2447-3359$

https://doi.org/10.21680/2447-3359.2021v7n1ID21228

\title{
OS RISCOS RELACIONADOS AO CARSTE NO NORDESTE BRASILEIRO
}

\section{Caio Lima dos Santos ${ }^{1}$; Saulo Roberto de Oliveira Vital ${ }^{2}$}

${ }^{1}$ Doutor em Geografia, Departamento de Geografia, Campus III, Universidade Estadual da Paraíba, Guarabira/PB, Brasil.

ORCID: https://orcid.org/0000-0002-6522-2603

Email: caiolimageo@gmail.com

${ }^{2}$ Doutor em Geociências, Departamento de Geociências, Centro de Exatas e da Natureza, Universidade Federa da Paraíba, João Pessoa/PB, Brasil

ORCID: https://orcid.org/0000-0002-2028-0033

E-mail: srovital@gmail.com

\section{Resumo}

O presente manuscrito constitui uma abordagem teórica sobre os riscos relacionados ao ambiente carstico no Nordeste Brasileiro, discutido no âmbito de uma mesa redonda na ocasião do I Simpósio de Redução de Riscos e Desastres, ocorrido entre os dias 05 e 08 de novembro 2019, promovido pelo Grupo de Pesquisa em Gerenciamento dos Riscos e Desastres Naturais (GENAT), da Universidade Federal do Rio Grande do Norte. Destarte, foram abordados a exploração das águas subterrâneas e a poluição dos aquíferos carsticos como principais fatores de riscos nesses ambientes, no contexto do Noreste do Brasil, além dos elementos relacionados a ocupação inadequada, assim como ocorre em outros ambientes. A base teórica para essa discussão partiu da análise de pesquisas que abordam essa temática na referida região geográfica brasileira.

Palavras-chave: Risco; Ambiente carstico; Nordeste do Brasil.

\section{THE RISKS RELATED TO KARST IN NORTHEAST BRAZIL}

\section{Abstract}

The present manuscript constitutes a theoretical approach on the risks related to the karst environment in Northeastern Brazil, discussed in the context of a round table on the occasion of the 1st Symposium on Risk and Disaster Reduction, which took place between the 5th and 8th of November 2019, promoted by research group on risk and disaster management (GENAT), from the Federal University of Rio Grande do Norte. The objective was to address the risks related to karst areas in Northeast Brazil. The exploration of groundwater and pollution of karst aquifers were addressed as the main risk factors in these environments in the context of the Northeast of Brazil, in addition to elements related to inappropriate occupation, as occurs in other environments. The theoretical basis for this discussion came from the analysis of research that addresses this theme in the aforementioned Brazilian geographical region.

Keywords: Risk; Karst environment; Northeast Brazil.

\section{LOS RIESGOS RELACIONADOS CON EL CARSTE EN EL NORESTE DE BRASIL}

\section{Resumen}

El presente manuscrito constituye un enfoque teórico sobre los riesgos relacionados con el entorno kárstico en el noreste de Brasil, discutido en el contexto de una mesa redonda con motivo del 1er Simposio sobre reducción de riesgos y desastres, que tuvo lugar entre el 5 y el 8 de noviembre de 2019, promovido por grupo de investigación sobre gestión de riesgos y desastres (GENAT), de la Universidad Federal de Rio Grande do Norte. El objetivo era abordar los riesgos relacionados con las áreas kársticas en el noreste de Brasil. La exploración de las aguas subterráneas y la contaminación de los acuíferos kársticos se abordaron como los principales factores de riesgo en estos entornos en el contexto del noreste de Brasil, además de elementos relacionados con la ocupación inapropiada, como ocurre en otros entornos. La base teórica para esta discusión provino del análisis de la investigación que aborda este tema en la región geográfica brasileña antes mencionada.

Palabras-clave: Riesgo; ambiente de karst; noreste de Brasil.

\section{INTRODUÇÃO}

A ocupação inadequada da superfície terrestre tem se revelado em variadas modalidades de risco para os grupos humanos que desenvolvem suas atividades nesses espaços e estabelecem relação direta com a dinâmica físico-natural, resultando, quase sempre, em prejuízos materiais, além da recorrente perda de vidas.

A região Nordeste do Brasil não foge desse contexto, onde é possível identificar diversas situações, desde o meio urbano ao 
rural, em que se verifica relação onerosa entre sociedade e natureza. Uma dessas modalidades de risco está associada ao contexto carstico, que, no caso da área analisada, ocorre em ambiente úmido, como também em ambiente semiárido.

Desse modo, a presente pesquisa tem como objetivo abordar o contexto ambiental observado nas áreas carsticas no Nordeste Brasileiro, no tocante aos riscos. Tal abordagem ganha fundamental importância na discussão acerca da geomorfologia antrópica, tendo em vista os seguintes aspectos: recente avanço da urbanização sobre áreas carsticas; a importância desses ambientes para a manutenção da vida humana, representada pelo fornecimento de água pelos aquíferos carsticos, sobretudo em regiões semiáridas; além da ausência de ações por parte do poder público, que visem orientar a ocupação dessas áreas, tendo como foco a mitigação dos riscos e a melhor adequação da ocupação humana.

Tal análise foi debatida em mesa redonda durante o I Simpósio de Redução de Riscos e Desastres, ocorrido em novembro de 2019, na Universidade Federal do Rio Grande do Norte, na Cidade de Caicó-RN. O referido evento cientifico contou com a participação de diversos agentes públicos, componentes de defesas civis de diferentes cidades da região Nordeste, onde foi possível chamar a atenção desses atores sociais para a relevância dos fenômenos carsticos na dinâmica ambiental, e, consequentemente, na deflagração de quadros de suscetibilidade, que por seu turno, podem se materializar em situações de risco, dando origem a situações de desastre.

Esse espaço possibilitou trazer para o debate ambiental, um tema que, recorrentemente, é negligenciado nas análises de risco. No nordeste, algumas poucas iniciativas a esse respeito foram empreendidas, tais como aquelas existentes em Maia et al. (2010), quando trata do processo de subsidência no município de LapãoBA, e em Vital e Travassos (2015), a respeito do carste subjacente da Bacia da Paraíba. A baixa quantidade de estudos pode estar relacionada à reduzida possibilidade de visualização dos fenômenos carsticos, uma vez que, embora tenham repercussões em superfície, os processos originários são difíceis de serem viasualizados, pois ocorrem em subsuperfície. Além disso, os desastres relacionados ao processo de carstificação, pelo menos no nordeste brasileiro, são muito raros, o que, de certo modo, gera uma certa despreocupação por parte da sociedade e dos gestores públicos. Sabe-se, portanto, que, nos países subdesenvolvidos, as políticas de prevenção do risco são muito incipientes, havendo uma maior atenção para ações de remediação, ou seja, medidas que são tomadas após a ocorrência do desastre. Destarte, o presente trabalho empreende um esforço em romper a falta de atenção frente a áleas, até então, pouco conhecidas, tal como a do carste.

\section{METODOLOGIA}

Este trabalho constitui uma pesquisa de cunho teórico, tendo como base a pesquisa bibliográfica em plataformas de pesquisas acadêmicas, onde foram selecionados artigos e teses desenvolvidas por diferentes autores que abordam os riscos em ambientes carsticos no contexto da Região Nordeste do Brasil.

Também foi desenvolvido um mapa representando as áreas de ocorrência de cavernas e as respectivas litologias superficiais associadas. O mesmo foi elaborado com base em dados do Centro
Nacional de Pesquisa e Conservação de Cavernas - CECAV (2018).

Foi utilizado arquivo em formato shape contendo os dados vetoriais disponibilizado pelo site do Instituto Chico Mendes ICMBio. Esses dados foram elaborados para todo o território brasileiro. Desse modo, os mesmos foram recortados com auxílio de software de geoprocessamento, utilizando como base dados da delimitação territorial da região Nordeste e dos seus respectivos estados em formato shape disponibilizado pelo Instituto Brasileiro de Geografia e Estatística (IBGE).

\section{RESULTADOS E DISCUSSÃO}

\subsection{Risco em Ambiente Carstico}

Os riscos em áreas carsticas, por vezes, são originados direta ou indiretamente por ações antrópicas. Desse modo, impactos causados podem ter consequências imprevisíveis devido as peculiaridades características desse ambiente.

A maior dificuldade para o estudo de áreas carsticas está na atuação de processos ativos em subsuperfície, controlados pela água, que, na maioria das vezes, não são diretamente observáveis, o que confere aos processos carsticos um caráter oculto, não despertando, assim, tanta atenção da sociedade (GUTIÉRREZ et al., 2014). O caráter oculto da rede drenagem no ambiente carstico torna extremamente difícil a previsão de resposta do

sistema as alterações induzidas pelo homem. Além disso, o sistema carstico apresenta alta sensibilidade dos elementos geomorfológicos e hidrológicos que o compõem frente às alterações na paisagem (DE WAELE et al., 2011). No entanto, a dinâmica desses ambientes pode ser identificada na superfície e suas evidências superficiais devem ser consideradas no processo de desenvolvimento urbano (PUEYO ANCHUELA et al., 2015).

Fenômenos como o colapso, a subsidência do solo, o alagamento e a inundação estão ligados a evolução de um carste, podendo trazer riscos para a população que ocupa essas áreas (LOLCAMA, 2002; MARÉCHAL et al., 2008).

A ocupação irregular em áreas cársticas resulta em impactos que se materializam notadamente na mudança do regime hidrológico superficial e subsuperficial, decorrente, sobretudo, da impermeabilização do solo e do soterramento de dolinas e pequenas depressões; vibrações oriundas do sobrepeso a partir da construção de equipamentos urbanos, responsáveis por acelerar a deflagração de subsidência e colapso da superfície; bem como o problema da poluição do aquífero (VESTENA et al., 2002). De acordo com Pereira (1998), o rebaixamento do nível da água pode provocar a perda do suporte hidrostático, que, assim como o sobrepeso de edificações no teto de cavidades carsticas, podem ocasionar a subsidência do terreno, configurando um fator de risco para a população residente.

As dolinas podem sofrer forte processo de alteração de sua morfologia decorrente de processos naturais ou por ação antrópica (Gutiérrez et al., 2011). O mesmo autor destaca ainda que a construção de edificações sobre essas feições alteradas pode refletir na ocorrência de danos a sociedade, pois as modificações ocorridas em feições preexistentes podem conduzir ao entendimento dessas áreas como estáveis do ponto de vista dos riscos, como é o caso de depressões soterradas no processo de urbanização. 
O rebaixamento do nível d'água decorrente da extração de água subterrânea é o principal fator responsável pela subsidência e colapso da superfície a partir da evolução de cavidades originadas na zona saturada da camada sedimentar que envolve o carste (VESTENA et al., 2002).

Desse modo, é fundamental agregar o máximo de dados geoambientais e socioambientais das áreas cársticas, no intuito de compreender sua dinâmica físico-natural e a interferência antrópica na deflagração de situações de risco, para mitigar os impactos produzidos nessa relação sociedade/natureza.

\subsection{Riscos em Ambientes Carsticos no Nordeste do Brasil}

$\mathrm{Na}$ região Nordeste do Brasil as áreas de ocorrência de processos carsticos estão localizadas com mais evidência nos estados da Bahia, Rio Grande do Norte e Ceará, com ocorrências mais pontuais e específicas no Piauí e Paraíba. Nesse contexto, os processos cársticos não ocorrem tão somente nos locais onde rochas carbonáticas afloram na superfície, como observa a geomorfologia carstica tradicional, mas se associa, também, a ambientes onde a rocha carbonática está recoberta por camadas de material não-carbonático, ou, até mesmo, onde há ausência de carbonatos.

A análise do contexto geológico da referida região geográfica brasileira demonstra que as características litológicas por si só não representam um fator determinante para a elaboração de morfologias cársticas, uma vez que a percolação de fluídos, potencializada pela porosidade secundária das rochas, é o gatilho comum para o desenvolvimento dos processos cársticos. A porosidade secundária, por sua vez, resulta das condições estruturais que caracterizam a geologia local. Desse modo, na referida região, são observadas situações onde o carste se desenvolve em superfície (carste exposto), e situações onde o carste se desenvolve em subsuperfície (carste subjacente), recoberto por camada de material rochoso não-carbonático.

A Figura 1 apresenta a ocorrência de cavernas na região Nordeste do Brasil. É importante destacar que essa feição, assim como outras morfologias do relevo carstico ocorre em diferentes litologias, com maior ênfase para rochas carbonáticas.

No estado da Bahia, observa-se a elaboração de relevo cárstico no contexto geológico da Formação Salitre, que, por sua vez, constitui uma plataforma carbonática de idade Proterozóica, componente do Grupo Una, Bacia de Irecê (KUCHENBECKER et al., 2011). Na referida área os problemas relacionados aos riscos em ambiente carstico estão demasiadamente ligados ao uso da água disponível no aquífero carstico, havendo, desde problemas de salinização nas áreas semiáridas, a riscos de subsidência e colapso do solo devido a exploração excessiva da água subterrânea.

Salles et al (2018) identifica no município de Iraquara (BA) situações de risco de subsidência e colapso do solo associados a evolução natural do sistema cárstico, uma vez que a área está sob domínio de rochas carbonáticas (dolimitos e calcários) da Formação Salitre, além da forte atuação das condições estruturais, orientando a gênese e evolução do relevo. Segundo esse autor as áreas de maior concentração de lineamentos são exatamente aquelas onde existe maior adensamento de dolinas, devido, sobretudo, à porosidade secundária, ou seja, esses são os locais de maior suscetibilidade a ocorrência de novos processos de dolinamento e onde os processos atuais apresentam maior relevância.

Desse modo, a expansão urbana e a exploração dos recursos hídricos nesse local podem acelerar os processos de subsidência e colapso do solo devido a perca da estabilidade hidrostática, constituindo um fator de risco nessa área.

Nos estados do Ceará e Rio Grande do Norte o ambiente cárstico está associado ao contexto geológico da Formação Jandaíra, que é constituída por litologia carbonática, havendo a ocorrência de cruzamento de estruturas lineares que se desenvolvem preferencialmente nas direções NE-SW e NW-SE. Situações de risco devido aos processos de subsidência e colapso foram identificadas com mais evidencia no campo de exploração denominado Fazenda Belém, que, por seu turno, constitui uma área de exploração de petróleo localizada no estado do Ceará, próximo à divisa com o estado do Rio Grande do Norte (XAVIER NETO, 2006).

Conforme Xavier Neto (2006) o surgimento de dolinas devido aos processos de subsidência e colapso do solo (Figura 2) está relacionado a pequena espessura da cobertura sedimentar, que se sobrepõe a rocha carbonática e ao preenchimento de fraturas por esse material, que facilmente pode ser suprimido e transportado para o interior das cavidades mediante a perca da estabilidade hidrostática do solo. Outros fatores podem potencializar esse processo, como a ocorrência de vibrações no solo devido a execução de obras de engenharia, bem como alterações na drenagem.

Em Teresina, capital do estado do Piauí observa-se a ocorrência de eventos relacionados a dinâmica do relevo cársticos, que resultam em situações de risco. A geologia local indica alta suscetibilidade a carstificação, mediante a junção de fatores como a ocorrência de rochas carbonáticas (calcários e dolimitos da Formação Pedra de Fogo) fraturadas decorrentes de esforços tectônicos. Somasse a esses fatores os impactos oriundos da dinâmica antrópica, revelada nos diferentes tipos de uso e ocupação do solo, além do desenvolvimento de atividades econômicas que podem potencializar os processos naturais.

Barradas et al. (2010) descreve eventos de subsidência e colapso do solo ocorridos nos anos de 1999 e 2008 na área central do município de Teresina. Na ocasião, cavidades se abriram na superfície, comprometendo as estruturas urbanas sobrepostas (Figura 3). Tais eventos estão relacionados, conforme os autores, a exploração excessiva de água subterrânea na área e a injeção de fluidos oriundos de rompimentos de galerias pluviais. Nesses pesquisa cabe salientar relatos de técnicos de empresas de perfuração de poços para o abastecimento hídrico sobre perca de equipamentos por afundamento, abertura de cavidades no solo, desaparecimento de água de piscinas, dentre outros, durante a abertura de novos poços tabulares. Essa aspectos demonstra a existência de um carste ativo, cujos vazios subterrâneos se desenvolvem próximo a superfície, fator que coloca o referido município numa situação de elevado risco associado aos processos carsticos. 


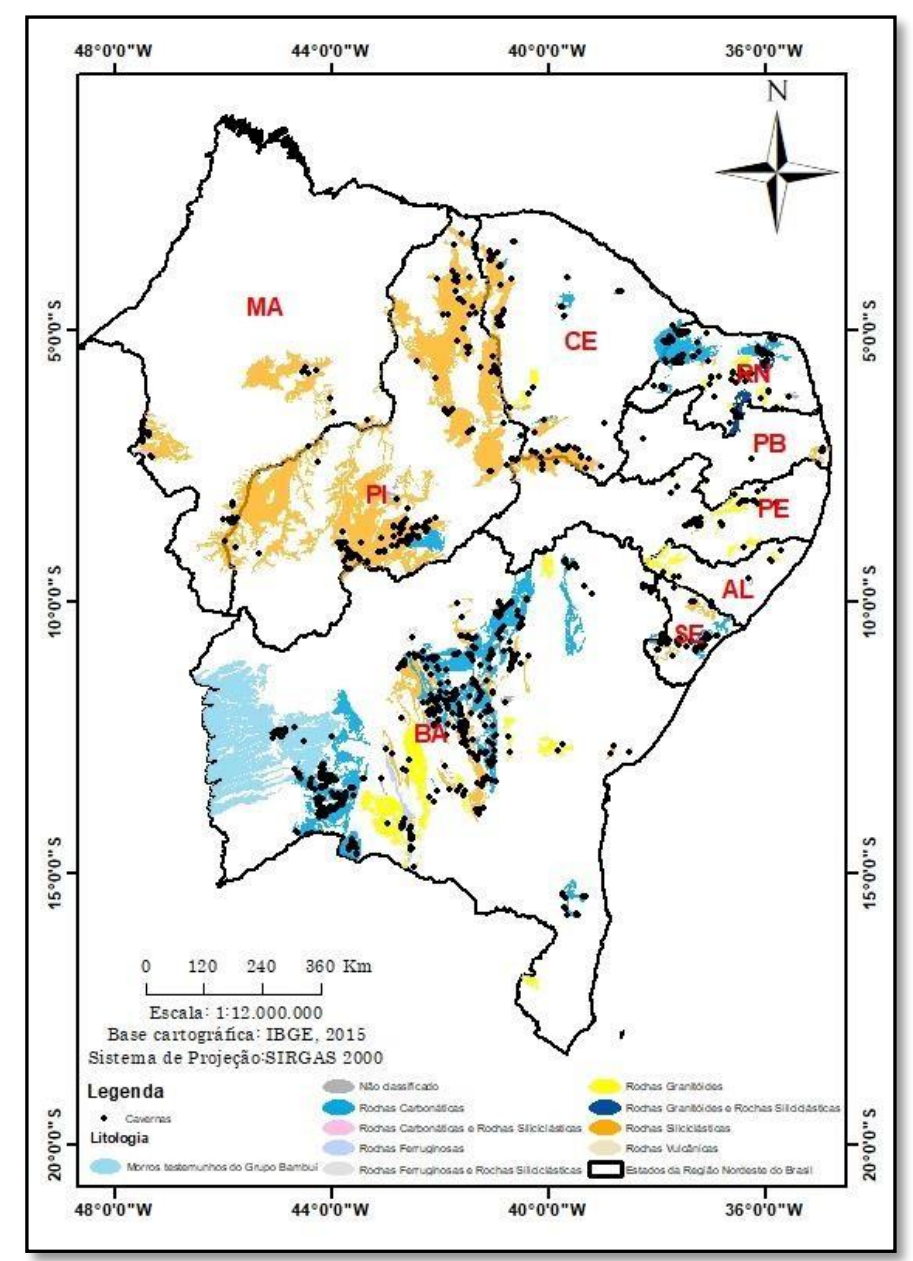

Figura 1 - áreas de ocorrência de caverna na região Nordeste do Brasil e associação litológica superficial. Fonte: modificado de CECAV (2018).
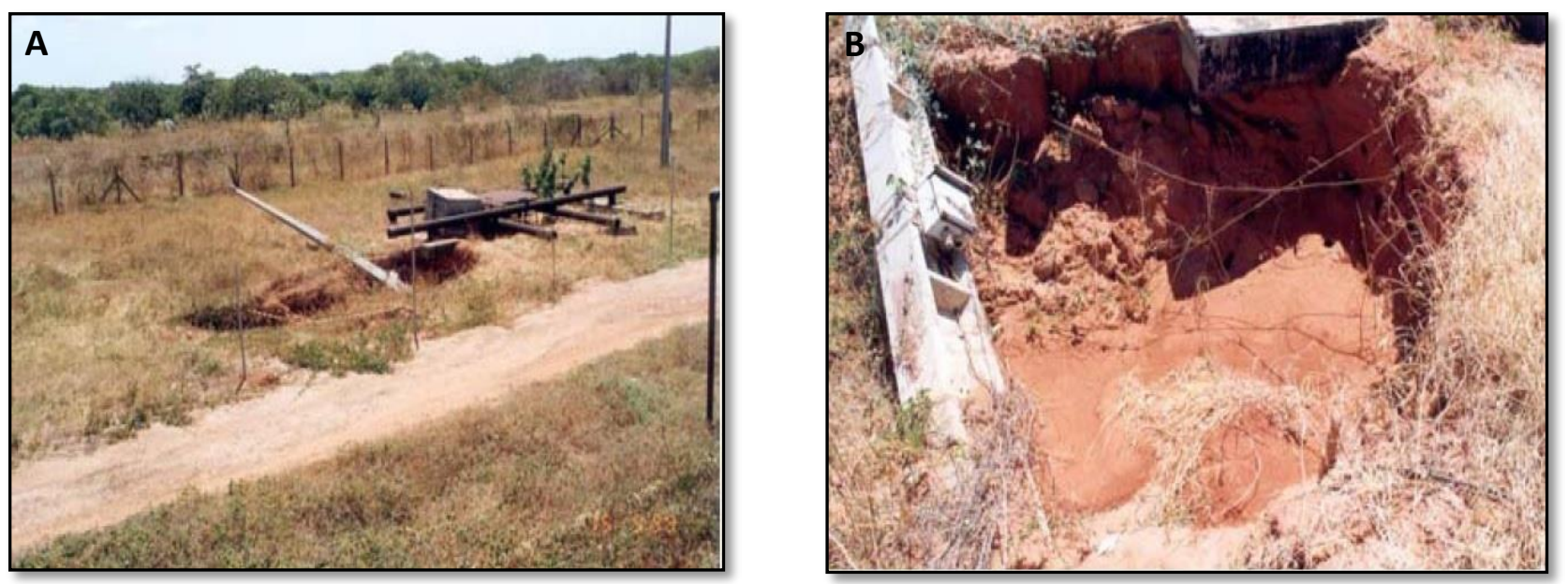

Figura 2 - a) e b) colapso do terreno em Fazenda Belém. Fonte: Xavier Neto (2006). 


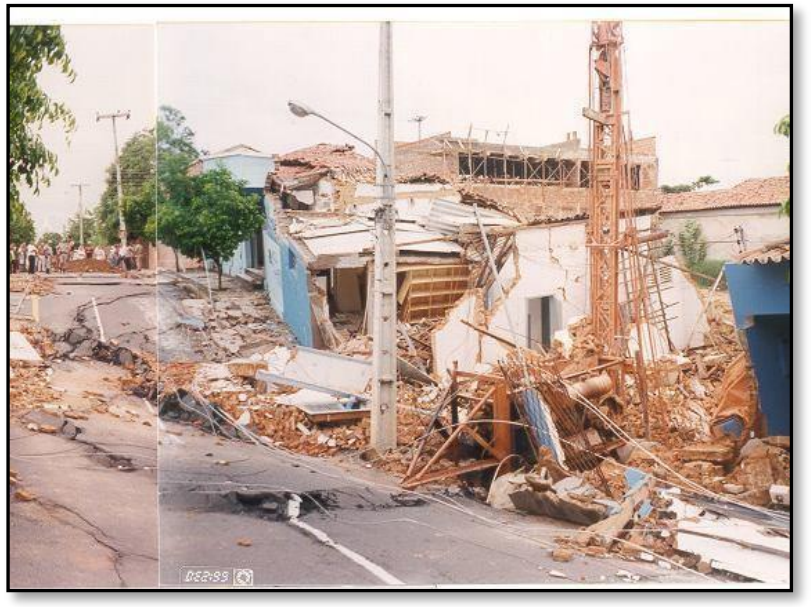

Figura 3 - evento de colapso do solo no município de Teresina/PI ocorrido no ano de 1999. Fonte: Pimentel (2008).

No estado da Paraíba a ocorrência do sistema cárstico está associada ao domínio geológico da Bacia Sedimentar Paraíba, que, dentro de seus limites, apresenta estratigrafia disposta em camadas de rochas de origem marinha (Calcário da Formação Gramame) e terrígenas (arenitos da formação Beberibe/Itamaracá). Recobrindo essas camadas, estão os sedimentos da Formação Barreiras, formada predominantemente por arenosos e argilosos, que formam uma espessa camada capeadora sobre as rochas carbonáticas (BARBOSA, 2007).

$\mathrm{Na}$ bacia sedimentar Paraíba, o calcário da Formação Gramame apresenta-se como o principal tipo de rocha a apresentar maior suscetibilidade ao processo carstificação, que, associada aos aspectos estruturais, representam o fator natural responsável pela elaboração do relevo carstico, representado com maior evidência pelas dolinas e depressões (VITAL, 2015).

As situações de risco relacionadas aos processos morfodinâmicos atuantes na elaboração do relevo carstico são observadas com mais evidência na região metropolitana de João Pessoa. Devido à ocupação urbana, diversas alterações superficiais foram impetradas na paisagem, provocando respostas ao cotidiano social. No entanto, não se tem atualmente conhecimento de nenhum mecanismo advindo do poder público para a gestão do território que faça qualquer menção as situações de riscos que a dinâmica do ambiente carstico pode causar, ou seja, a ocupação do espaço se dá sem considerar esse aspecto litológico e a morfodinâmica de tal ambiente. No que se refere às pesquisas acadêmicas, foram registradas algumas iniciativas, tais como aquelas existentes em Furrier e Vital (2011), Vital (2015), Vital e Travassos (2015) e Vital et al. (2020).

A baixa quantidade de estudos que tratam dos riscos na cidade de João Pessoa/PB pode estar relacionado à ausência de registros de ocorrências relacionadas a problemas oriundos de eventos associados ao relevo carstico, ou mesmo, pelo não reconhecimento desse tipo de processo numa área recoberta. Situações recorrentes na cidade de João Pessoa/PB como a abertura de crateras (Figura 4a) são tratados como simples resultado do estouro de tubulação pluvial; a ocorrência de rachaduras nas paredes de edificações é considerada como problemas nas estruturas prediais (figura 04b); as inundações e os recorrentes casos de alagamento são tratados como deficiência na drenagem urbana (Figura 4c); assim, não se considera a importância das dolinas como ponto de entrada de água para o lençol freático, de modo que se destina para elas todo tipo de efluentes produzidos pelas atividades humanas (Figura 04d).

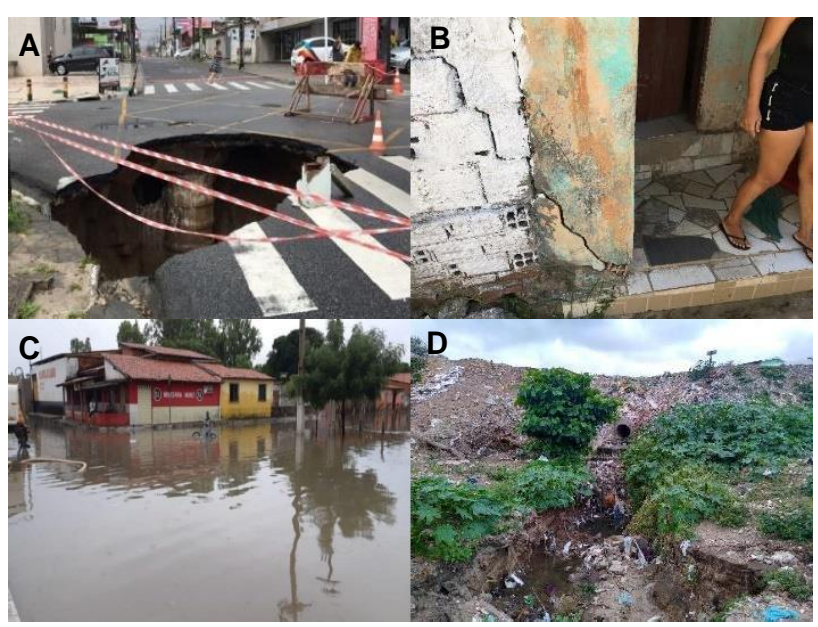

Figura 4 - a) cavidade gerado por processo remoção mecânica de sedimentos decorrente da injeção de fluxo subterrâneo devido o estouro de galeria pluvial após atuação de sistema de climático que provocou chuvas intensas na área no ano de 2017; b) rechadura observada em paredes de residência edificada dentro de uma depressão cárstica localizada no bairro João Paulo II; c) registro de evento de enchente ocorrida em depressão cárstica no bairro Jardim Veneza. Fonte: Informa Paraíba (2019); d) disposição de efluentes para o interior de uma depressão carstica localizada no bairro Cidade dos Funcionários I.

Como é corriqueiro acontecer no Brasil, os problemas de ordem ambiental só despertam interesse na sociedade e, consequentemente no poder público, quando se revelam em forma de catástrofe, levando a perca de vidas humanas e de bens materiais. É preciso compreender que os fenômenos da natureza ocorrem numa escala de tempo distinto da escala de vida humana, fazendo com que ao longo de uma vida, um indivíduo ou grupo social não consigam, por vezes, observar quaisquer mudanças significativas numa paisagem carstica que, apesar de dinâmicas processuais naturais relativamente lentas, podem apresentar processos abruptos. No entanto, as intervenções antrópicas podem alterar a dinâmica natural destes ambientes, conduzindo ao desencadeamento ou intensificação de processos de diversas ordens.

Portanto, o reconhecimento das condições de risco que possam atingir o cotidiano das pessoas é de grande importância para orientar o uso e ocupação da superfície terrestre e minimizar a ocorrência de problemas que potencializam e/ou deflagram os fenômenos naturais. 


\section{CONSIDERAÇÕES FINAIS}

A maior dificuldade de abordagem em considerar os riscos relacionados a processos cársticos está, dentre outros aspectos, na dificuldade de visualizar os processos (dissolução) e a atuação dos fatores deflagradores (caracterização estrutural, percolação de fluídos nas rochas, etc). Outro aspecto relevante é a baixa recorrência de fenômenos geradores de desastres, quando comparado aos fenômenos hidrológicos e erosivos, por exemplo, dando a ideia de aparente estabilidade ambiental.

O principal fator de risco em ambientes carsticos no Nordeste do Brasil observado nessa análise está relacionado a exploração da água acumulada nos vazios subterrâneos elaborados pela dissolução da rocha cárstica, além do problema da poluição desses aquíferos a partir da injeção de fluídos gerados por atividades antrópicas.

Portanto, reafirma-se a fundamental importância dos ambientes carsticos para a manutenção da vida humana, sobretudo nas zonas secas, além do risco gerado pela inobservância dos processos naturais característicos desse ambiente no processo de uso e ocupação da superfície terrestre.

\section{REFERÊNCIAS}

BARBOSA, J. A. A deposição carbonática na faixa costeira Recife-Natal: aspectos estratigráficos, geoquímicos $e$ paleontológicos. 270f. Tese (Doutorado). Programa de PósGraduação em Geociências. Universidade Federal de Pernambuco, 2007.

BARRADAS, M. T. GOMES, E. CARVALHO, A. VIEIRA, C. I. Mapeamento dos colapsos e subsidências do solo em Teresina - PI. In: Anais V Congresso Norte-Nordeste de Pesquisa e Inovação. Maceió: IFAL, 2010.

BRASIL. Serviço Geológico do Brasil. CPRM. Estudos sobre a instabilidade do terreno nos bairros Pinheiro, Mutange e Bebedouro, Maceió (AL): volume I, relatório síntese dos resultados $n$. 1. CPRM, 2019.

CECAV - Centro Nacional de Pesquisa e Conservação de Cavernas. Áreas de ocorrência de cavernas no Brasil, 2018. Instituto Chico Mendes. Disponível em: https://www.icmbio.gov.br/cecav/projetos-eatividades/provinciasespeleologicas.html Acesso em $15 / 04 / 2020$

DE WAELE, J. GUTIÉRREZ, F. PARISE, M. PLAN, L. Geomorphology and natural hazards in karst areas: a review. In: Geomorphology, v. 134, n. 1-2, p. 1-8, 2011.

GUTIÉRREZ, T. PARISE, M. DE WAELE, J. GUTIÉRREZ, F. GALVE, J. P. LUCHA, P. CASTAÑEDA, C. BONACHEA, J. GUERRERO, J. Integrating geomorphological mapping, trenching, InSAR and GPR for the identification and characterization of sinkholes in the mantled evaporite karst of the Ebro Valley (NE Spain). In: Geomorphology, n. 134, 144-156, 2011.
GUTIÉRREZ, T. PARISE, M. DE WAELE, J. JOURDE, H. A review on natural and human-induced geohazards and impacts in karst. In: Earth-Science Reviews, v. 138, p. 6188, 2014.

INFORMA PARAÍBA. Disponível em <https://informaparaiba.com.br/2019/07/15/mppb-cobrapolitica-publica-para-os-moradores-da-comunidade-treslagoas-que-foram-afetados-por-enchentes/> Acesso em 17/08/2020.

KUCHENBECKER, M. REIS, H. L. S. FRAGOSO, D. G. C. Caracterização estrutural e considerações sobre a evolução tectônica da Formação Salitre na porção central da Bacia de Irecê, norte do Cráton do São Francisco (BA). Geonomos, v. 19, n.2, 42-49, 2011.

LOLCAMA, J. L. COHEN, H. A. TONKIN, M. J. Deep karst conduits, flooding, and sinkholes: lessons for the aggregates industry. In: Engineering Geology, v. 65, n. 2-3, p. 151-157, 2002.

MAIA, P. H. P.; DIAS-NETO, B.; CORRÊA-GOMES, L. C. A análise geológica e estrutural das fissuras e subsidências no carste Lapão. Cadernos de Geociências, v. 7, n. 2, 2010.

MARÉCHAL, J. C. LADOUCHE, B. DÖRFLIGER, N. Karst flash flooding in a Mediterranean karst, the example of Fontaine de Nîmes. In: Engineering Geology, v. 99, n. 3-4, p. 138-146, 2008.

PEREIRA, R. G. F. A. Caracterização Geomorfológica e Geoespelelógica do Carste da Bacia do Rio Una, Borda Leste da Chapada Diamantina (Município de Itaetê, Estado da Bahia). Dissertação de Mestrado. Instituto de Geociências USP, 1998.

PIMENTEL, J. Contribuição para o Entendimento dos Processos de Colapso e Subsidência de Solo em Teresina. CPRM Serviço Geológico do Brasil - DEGET/DHT, 2008.

PUEYO ANCHUELA, Ó. CASAS SAINZ, A. M. POCOVÍ JUAN, A. GIL GARBÍ, H. Assessing karst hazards in urbanized areas. Case study and methodological considerations in the mantle karst from Zaragoza city (NE Spain). In: Engineering Geology, № 184, p. 29-42, 2015.

SALLES, L. Q. Hidrogeologia e risco geológico em rochas carbonáticas proterozoicas: porção central da Chapada Diamantina, Bahia, Brasil. 87f. Dissertação (Mestrado em Geologia). Programa de Pesquisa e Pós-graduação em Geologia, Universidade Federal da Bahia, Salvador, 2017.

SALLES, L. Q. GALVÃO, P. LEAL, L. R. B. PEREIRA, R. G. F. A. PURIFICAÇÃO, C. G. C. LAUREANO, F. V. Evaluation of susceptibility for terrain collapse and subsidence in karst areas, municipality of Iraquara, Chapada Diamantina (BA), Brazil. Environmental Earth Sciences, 77(16), 593, 2018. 
VITAL, S. R. O. Análise geológica-geomorfológica das depressões fechadas e dolinas em sedimentos da Formação Barreiras na região de João Pessoa (PB). 216f. Tese (Doutorado em Geologia) - Programa de Pós-graduação em Geociências, Centro de Tecnologia e Geociências, Universidade Federal de Pernambuco, Recife, PE, 2015.

VESTENA, L. R. KOBIYAMA, M. SANTOS, J. C. Considerações sobre gestão ambiental em áreas cársticas. In: $R A^{\prime} E G A$, p. 81-94, 2002.

VITAL, S. R. O.; TRAVASSOS, L. E. P. Impactos decorrentes do uso do solo em dolinas da Bacia Sedimentar da Paraíba, zona oeste do município de João Pessoa (PB), Brasil. Caderno de Geografia, v. 25, n. 4, p. 118-133, 2015.

VITAL, S. R. O.; BARRETO, A. M. F.; SALLUN-FILHO, W.; TRAVASSOS, L. E. P.; PINHO, I. G. Gênese e Evolução de Depressões Fechadas na Bacia da Paraíba. William Morris Davis - Revista de Geomorfologia, Sobral, n. 2, [2020?]. No prelo.
XAVIER NETO, P. Processamento e interpretação de dados 2D e 3D de GPR: Aplicações no imageamento de feições Kársticas e estruturas de dissolução no campo de petróleo de Fazenda Belém - CE. Tese (Doutorado em Geologia). Programa de graduação em Geodinâmica e Geofísica, Universidade Federal do Rio Grande do Norte, Natal-RN, 2006.

\section{AGRADECIMENTOS}

Os autores agradecem aos organizadores do I Simpósio de Redução de Riscos e Desastres pelo espaço aberto para o debate sobre temas relevantes ao desenvolvimento social.

Recebido em: 04/06/2020

Aceito para publicação em: 18/05/2021 